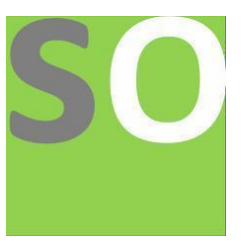

Article title: A Note Concerning Fermat Numbers

Authors: Monica Feliksiak [1]

Affiliations: N.A.[1]

Orcid ids: 0000-0002-5786-4227[1]

Contact e-mail: monicafeliksiak@yahoo.com

License information: This work has been published open access under Creative Commons Attribution License http://creativecommons.org/licenses/by/4.0/, which permits unrestricted use, distribution, and reproduction in any medium, provided the original work is properly cited. Conditions, terms of use and publishing policy can be found at https://www.scienceopen.com/.

Preprint statement: This article is a preprint and has not been peer-reviewed, under consideration and submitted to ScienceOpen Preprints for open peer review.

DOI: 10.14293/S2199-1006.1.SOR-.PPNFYON.v1

Preprint first posted online: 06 September 2021

Keywords: Fermat numbers, Structure of Fermat numbers. 


\title{
A NOTE CONCERNING FERMAT NUMBERS
}

\author{
MONICA U. FELIKSIAK
}

\begin{abstract}
The Fermat's Numbers are clearly all of the form $6 n+5$, with one exception to this rule. This paper presents a general formula, showing the structure of Fermat numbers.
\end{abstract}

2010 Mathematics Subject Classification. 11A41, 11A51, 11D09, 1102.

Key words and phrases. Fermat numbers, structure of Fermat numbers. 


\section{FERMAT Numbers}

Fermat Numbers are positive integers of the form:

$$
\mathbf{F}_{s}=2^{2^{s}}+1
$$

Pierre de Fermat while studying the Fermat numbers conjectured that all these numbers are prime. Leonhard Euler however, in 1732 demonstrated that $\mathbf{F}_{5}$ is composite. Currently, there are only 5 Fermat primes known, it is widely believed that these are the only Fermat primes. Factoring Fermat numbers is hard, due to the sheer size of those numbers. The numbers are of the form $6 n+5$, with a single exception - the first Fermat number. We would like to analyze the structure of those numbers from this point of view. First, we make some pertinent definitions:

Definition 0.1. $\mathbf{F}_{0}=2^{2^{0}}+1=6(0)+3$

Definition 0.2. $\mathbf{F}_{1}=2^{2^{1}}+1=6(0)+5$

Definition 0.3. $\mathbf{F}_{2}=2^{2^{2}}+1=6\left(2^{\left(2^{1}-1\right)}\right)+5$

Subsequent Fermat numbers are defined by a formula. First we need to establish an auxiliary formula:

Lemma 0.4 (Auxiliary product).

The auxiliary product for all $m \in \mathbb{N} \mid m \geq 3$ is given by:

$$
3 \times \prod_{(k=1)}^{(m-2)}\left(2\left(2^{\left(2^{k}-1\right)}\right)+1\right)=2^{\left(2^{(m-1)}\right)}-1
$$

Proof.

Clearly, the formula 0.1 holds for $m=3$ as it produces:

$$
3 \times 5=2^{2^{2}}-1
$$

Suppose that the formula holds for some $(m-1) \in \mathbb{N} \mid m>3$. Then,

$$
\left(2^{2^{m}}-1\right)=\left(2^{2^{(m-1)}}-1\right)+\left(2^{2^{(m-1)}}\right)\left(2^{2^{(m-1)}}-1\right)
$$

therefore,

$$
\begin{aligned}
\left(2^{2^{(m-1)}}-1\right)+ & \left(2^{2^{(m-1)}}\right)\left(2^{2^{(m-1)}}-1\right) \\
=3 \times 5 \times & \times 17 \times \ldots \times\left(2\left(2^{\left(2^{(m-2)}-1\right)}\right)+1\right) \\
& +\left(2^{2^{(m-1)}}\right)\left(3 \times 5 \times 17 \times \ldots \times\left(2\left(2^{\left(2^{(m-2)}-1\right)}\right)+1\right)\right)
\end{aligned}
$$

which is equal to:

$$
3 \times 5 \times 17 \times \ldots \times\left(2\left(2^{\left(2^{(m-2)}-1\right)}\right)+1\right)\left(2\left(2^{\left(2^{(m-1)}-1\right)}\right)+1\right)=\left(2^{2^{m}}-1\right)
$$

Consequently by the principle of mathematical induction, the formula 0.1 holds $\forall m \in \mathbb{N} \mid m \geq 3$. Consequently, Lemma 0.4 holds for all $m \in \mathbb{N} \mid m \geq 3$, concluding the proof.

Now the formula for the subsequent Fermat numbers. 
Theorem 0.5 (Fermat numbers).

Subsequent, (apart from the three defined above) Fermat numbers are given by:

$$
\begin{aligned}
\mathbf{F}_{s}=2^{2^{s}} & +1=6 n_{s}+5 \quad \text { where: } \\
& n_{s}=\left(2+\sum_{k=1}^{(s-2)}\left(2^{\left(2^{(k+1)}-1\right)}\right) \times \prod_{m=1}^{k}\left(2\left(2^{\left(2^{m}-1\right)}\right)+1\right)\right) \quad \forall s \in \mathbb{N} \mid s \geq 3
\end{aligned}
$$

Proof.

The formula 0.5 is clearly valid for $s=3$ since,

(0.6) $\quad \mathbf{F}_{3}=2^{2^{3}}+1=6 n_{3}+5=6\left(2+2^{\left(2^{2}-1\right)} \times\left(2\left(2^{\left(2^{1}-1\right)}\right)+1\right)\right)+5=257$

Suppose therefore, that the formula holds for some $(s-1) \in \mathbb{N} \mid(s-1) \geq 3$. Then,

$$
\mathbf{F} s=\left(\mathbf{F}_{(s-1)}-1\right)^{2}+1=\left(2^{2^{(s-1)}}\right)+\left(2^{2^{(s-1)}}\right)\left(2^{2^{(s-1)}}-1\right)+1
$$

Hence we obtain,

$$
\left(2^{2^{(s-1)}}\right)+1+\left(2^{2^{(s-1)}}\right)\left(2^{2^{(s-1)}}-1\right)=\left(6\left(n_{(s-1)}\right)+5\right)+2\left(2^{\left(2^{(s-1)}-1\right)}\right)\left(2^{2^{(s-1)}}-1\right)
$$

From 0.1 we have:

$$
2\left(2^{2^{(s-1)}}-1\right)=6\left(\prod_{(n=1)}^{(s-2)}\left(2\left(2^{\left(2^{n}-1\right)}\right)+1\right)\right)
$$

Hence,

$$
\text { (0.10) } 2\left(2^{\left(2^{(s-1)}-1\right)}\right)\left(2^{2^{(s-1)}}-1\right)=6\left(\left(2^{\left(2^{(s-1)}-1\right)}\right) \times \prod_{(n=1)}^{(s-2)}\left(2\left(2^{\left(2^{n}-1\right)}\right)+1\right)\right)
$$

and so,

$$
\begin{aligned}
& (6.11) \\
= & 6\left(2+\sum_{(k=1)}^{(s-3)} 2^{\left(2^{(k+1)}-1\right)} \times \prod_{(m=1)}^{k}\left(2\left(2^{\left(2^{m}-1\right)}\right)+1\right)\right)+\left(2^{\left(2^{(s-1)}-1\right)}\right) \times \prod_{(n=1)}^{(s-2)}\left(2\left(2^{\left(2^{n}-1\right)}\right)+1\right)+5 \\
= & 6\left(2+\sum_{(k=1)}^{(s-2)}\left(2^{\left(2^{(k+1)}-1\right)}\right) \times \prod_{(m=1)}^{k}\left(2\left(2^{\left(2^{m}-1\right)}\right)+1\right)\right)+5=6\left(n_{s}\right)+5=\mathbf{F}_{s}
\end{aligned}
$$

Hence by the principle of mathematical induction, the formula 0.5 holds for all $s \in \mathbb{N} \mid s \geq 3$, Consequently Theorem 0.5 holds for all $s \in \mathbb{N} \mid s \geq 3$, concluding the proof.

As an example Fermat number computation for $s=6$ :

\section{Example 0.6.}

$$
\begin{aligned}
& \mathbf{F}_{6}=2^{2^{6}}+1= \\
& \begin{aligned}
6 \times\left(2+5 \times 2^{\left(2^{2}-1\right)}+5 \times 17 \times 2^{\left(2^{3}-1\right)}+5 \times 17 \times 257 \times 2^{\left(2^{4}-1\right)}\right. \\
\left.+5 \times 17 \times 257 \times 65537 \times 2^{\left(2^{5}-1\right)}\right)+5
\end{aligned}
\end{aligned}
$$




\section{REFERENCES}

Chahal, Jasbir S. 1988. Topics in number theory, Plenum Press, New York.

Cohn, Harvey. 1980. Advanced number theory, Dover Publications, New York.

Ireland, Kenneth and Michael Rosen. 1990. A classical introduction to modern number theory, Springer Verlag, New York.

Rose, H.E. 1995. A course in number theory, Oxford Science Publications, Oxford.

Stopple, Jeffrey. 2003. A primer of analytic number theory, Cambrige University Press, Cambrige. 